Chinese Journal of Organic Chemistry

\title{
一氟甲基二硫化物的高效合成
}

\author{
胡晓光 ${ }^{a}$ 庞仁艺 ${ }^{a}$ 郑天骄 ${ }^{a}$ 姚瑞超 ${ }^{a}$ 陈文博 $*, a, b$ \\ $\left({ }^{a}\right.$ 上海电力大学环境与化学工程学院 上海市电力材料防护与新材料重点实验室 上海 200090) \\ $\left({ }^{b}\right.$ 中国科学院上海有机化学研究所 中科院能量调控材料重点实验室 上海 200032)
}

\begin{abstract}
摘要 以 $\mathrm{PhSO}_{2} \mathrm{SCH}_{2} \mathrm{~F}$ 为一氟甲硫基化试剂, 室温下实现了硫酚/硫醇的一氟甲硫基化反应, 高收率地合成了一系列一 氟甲基二硫化物. 该方法具有反应时间短、条件温和、操作简单以及无需外加添加剂等特点，为高效制备一氟甲基二 硫化物提供了一条简便的途径.
\end{abstract}

关键词 一氟甲硫基; 二硫化物; $\mathrm{PhSO}_{2} \mathrm{SCH}_{2} \mathrm{~F} ;$ 硫酚

\section{High Efficient Synthesis of Monofluoromethyldisulfides}

\author{
Hu, Xiaoguang ${ }^{a} \quad$ Pang, Renyi $^{a} \quad$ Zheng, Tianjiao $^{a} \quad$ Yao, Ruichao $^{a}$ Chen, Wenbo ${ }^{*, a, b}$
}

( ${ }^{a}$ Shanghai Key Laboratory of Materials Protection and Advanced Materials in Electric Power, College of Environmental and Chemical Engineering, Shanghai University of Electric Power, Shanghai 200090)

( ${ }^{b}$ CAS Key Laboratory of Energy Regulation Materials, Shanghai Institute of Organic Chemistry, Chinese Academy of Sciences, Shanghai 200032)

\begin{abstract}
The monofluoromethylthiolation reaction of thiophenols/mercaptans was developed at room temperature using $\mathrm{PhSO}_{2} \mathrm{SCH}_{2} \mathrm{~F}$ as monofluoromethylthiolation reagent, and a series of monofluoromethyl disulfides were synthesized in good yields. This method features short reaction time, mild conditions, simple operation and no additives. It provides a simple and convenient way for the efficient preparation of monofluoromethyl disulfides.
\end{abstract}

Keywords monofluoromethylthio; disulfide; $\mathrm{PhSO}_{2} \mathrm{SCH}_{2} \mathrm{~F}$; thiophenol

由于氟原子独特的氟效应，有机含氟化合物在农 药、医药、材料等领域扮演着重要的角色 ${ }^{[1]}$. 有机功能 分子中，在特定的位置引入氟原子或含氟基团成为一种 重要的策略来调控其物理和化学性质. 氟烷硫基 ${ }^{[2]}$ 如三 氟甲硫基 $\left(\mathrm{SCF}_{3}\right)$ 、二氟甲硫基 $\left(\mathrm{SCF}_{2} \mathrm{H}\right)$ 和一氟甲硫基 $\left(\mathrm{SCH}_{2} \mathrm{~F}\right)$, 作为一类重要的含氟基团, 因其优秀可调的 亲脂性和代谢稳定性吸引了药物化学家的极大研究兴 趣. 与三氟甲硫基 ${ }^{[3]}$ 和二氟甲硫基 ${ }^{[4]}$ 化合物的广泛研究 相比, 一氟甲硫基化合物的报道相对较少. 尽管报道不 多, 但已知的一氟甲硫基化合物展示了出色的生物活 性. 如图 1 所示, Fluticasone 及其衍生物 fluticasone propionate $^{[5]}$, Androstanes ${ }^{[6]}$, Sulindac ${ }^{[7]}$ 等是一类抗炎药 分子. 一氟甲硫基化合物在生物活性方面的巨大潜力, 吸引有机化学家合成各种各样一氟甲硫基化合物来满
足日益增长的药物化学需求. 近年来科研工作者发展了 一系列一氟甲硫基化试剂 ${ }^{[8]}$, 大大促进了一氟甲硫基化 合物的高效合成. 比如利用 $\mathrm{PhSO}_{2} \mathrm{SCH}_{2} \mathrm{~F}$ 作为一氟甲硫 基化试剂，可以实现对非活化烯烃、芳基硼酸、醛以及 亚磷酸酯的一氟甲硫基化反应，高效制备相应一氟甲硫 基的梄 ${ }^{[8]}$ 、羧酸酯 ${ }^{[9]}$ 以及磷酸酯 ${ }^{[10]}$ 类化合物(图 1).

二硫化物是一类重要的有机化合物, 分子中 $\mathrm{S}-\mathrm{S}$ 键具有特殊的生物化学特性, 在制药和食品化学中都有 着独特且不可替代的作用 ${ }^{[11]}$. 尤其是不对称的二硫化 物, $\mathrm{S}-\mathrm{S}$ 键两边各连接着一个不同的基团, 这使得药物 分子会拥有不同的药理结构 ${ }^{[12]}$, 从而具有十分优秀的 抗病毒特性，同时在一定程度上还能使得整个分子的稳 定性增强. 由于氟原子独特的氟效应，氟烷基二硫化合 物的研究有望为合成具有潜在的生物活性分子提供一

\footnotetext{
* Corresponding author. E-mail: wenbochen@shiep.edu.cn

Received September 1, 2020; revised November 2, 2020; published online November 27, 2020.

Project supported by the Natural Science Foundation of Shanghai (No. 20ZR1471600), the Science and Technology Commission of Shanghai Municipality (No. 19DZ2271100) and the Open Research Fund Program of CAS Key Laboratory Of Energy Regulation Materials (No. ORFP2020-06).

上海市自然科学基金(No. 20ZR1471600)、上海市科学技术委员会(No. 19DZ2271100)和中国科学院能量调控材料重点实验室开放基金(No. ORFP2020-06)资助项目.
} 


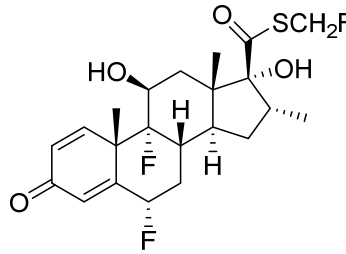

Fluticasone

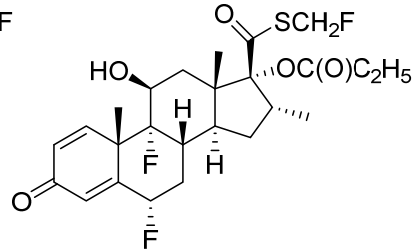

Fluticasone propionate

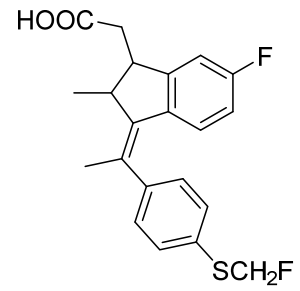

Sulindac

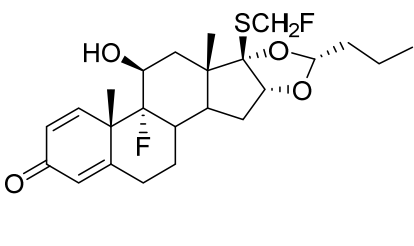

Androstanes
图 1 含有一氟甲硫基的活性分子

Figure 1 Bioactive molecules with monofluoromethylthio group

种新的思路. 与三氟甲基二硫化物 ${ }^{[13]}$ 和二氟甲基二硫 化物 ${ }^{[14]}$ 相比, 一氟甲基二硫化物研究相对较少. 易文斌 课题组 ${ }^{[\mathrm{b}]}$ 报道了使用他们课题组发展的一氟甲硫基 Bunte 盐 $\mathrm{CFH}_{2} \mathrm{SSO}_{3} \mathrm{Na}$ 作为一氟甲硫基化试剂, 以甲醇 为溶剂, 在 $50{ }^{\circ} \mathrm{C}$ 与各种取代苯硫酚反应 $12 \mathrm{~h}$, 以中等 的收率得到一系列一氟甲基二硫化物(Scheme 1, a).

(a) Yi's work<smiles>Sc1cc[R]cc1</smiles>

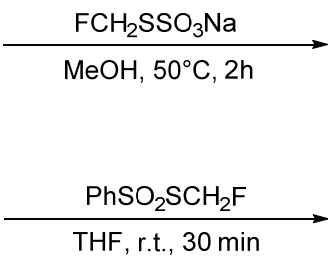

$\mathrm{R}-\mathrm{S}-\mathrm{SCH}_{2} \mathrm{~F}$

$58-78 \%$

(b) This work

$$
\begin{array}{ll}
\mathrm{R}-\mathrm{SH} & \mathrm{PhSO}_{2} \mathrm{SCH}_{2} \mathrm{~F} \\
\mathrm{THF}, \text { r.t., } 30 \mathrm{~min} & \mathrm{R}-\mathrm{S}-\mathrm{SCH}_{2} \mathrm{~F} \\
\text { up to } 96 \%
\end{array}
$$

图式 1 一氟甲基二硫化物的制备方法

Scheme 1 Methods for the preparation of monofluoromethyldisulfides

雷爱文课题组 ${ }^{[15]}$ 利用电化学氧化脱氢的方法, 通 过硫酚(硫醇)之间的交叉偶联来制备不对称二硫化合 物. 受该工作的启发, 我们小组希望用苯硫酚与 $\mathrm{PhSO}_{2}-$ $\mathrm{SCH}_{2} \mathrm{~F}$ 反应来制备一氟甲基二硫化物. 有趣的是, 控制 实验表明该反应在不通电的情况下也能顺利进行. 我们 以 $\mathrm{PhSO}_{2} \mathrm{SCH}_{2} \mathrm{~F}$ 作为一氟甲硫基化试剂和一系列硫酚或 硫醇反应, 在温和条件下高效制备了一氟甲基二硫化物 (Scheme 1, b).

\section{1 结果与讨论}

\section{1 反应条件篮选}

选择对氯苯硫酚作为反应底物, $\mathrm{PhSO}_{2} \mathrm{SCH}_{2} \mathrm{~F}$ 作为
一氟甲硫基化试剂，室温下对反应条件进行研究，结果 如表 1 所示. 首先, 尝试在二氯甲烷中对 4-氯苯硫酚和 $\mathrm{PhSO}_{2} \mathrm{SCH}_{2} \mathrm{~F}$ 的投料比进行优化, 当二者物质的量之比 为 $1.5: 1$ 时, 产物 3a 的分离收率为 $55 \%$, 同时观察到 反应中有明显的对氯苯硫酚自身偶联的二硫化物生成 (Entry 1). 当二者物质的量之比为 $1: 1$ 时, $\mathbf{3 a}$ 的收率增 加到 $81 \%$ (Entry 2); 当二者物质的量之比为 $1: 1.5$ 时, 反应收率为 $89 \%$, 对氯苯硫酚自身偶联产物明显得到抑 制(Entry 3). 随后对反应的溶剂进行了篮选，结果表明 该反应在大多数溶剂中都能够顺利进行(Entries $4 \sim 11$ ), 在四氢呋喃中有着最高收率为 $95 \%$ (Entry 4). 在极性溶 剂中，该反应收率有所降低，在二甲基亚砜(DMSO)中 收率为 79\% (Entry 10). 在 N,N-二甲基甲酰胺(DMF)中 收率为 66\% (Entry 11), 收率明显降低是由于对氯苯硫 酚自身偶联. 同时, 也考察了氧气对反应的影响, 结果 表明在氮气保护条件下, 该反应在四氢呋喃中的收率没 有明显变化(Entry 12). 最终, 选择 4-氯苯硫酚和 $\mathrm{PhSO}_{2} \mathrm{SCH}_{2} \mathrm{~F}$ 物质的量之比为 $1: 1.5$, 四氢呋喃为溶剂, 室温下反应，作为最优的反应条件.

表 1 反应条件筛选

Table 1 Screening of the reaction conditions

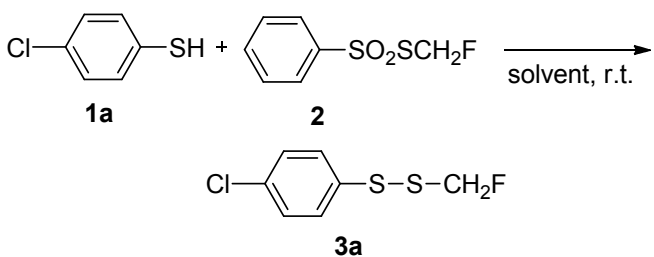

$3 a$

\begin{tabular}{cclc}
\hline Entry & $n(\mathbf{1 a}): n(\mathbf{2})$ & Solvent & Yield $^{a} \%$ \\
\hline 1 & $1.5: 1$ & DCM & 55 \\
2 & $1: 1$ & DCM & 81 \\
3 & $1: 1.5$ & DCM & 89 \\
4 & $1: 1.5$ & THF & 95 \\
5 & $1: 1.5$ & $\mathrm{CH}_{3} \mathrm{CN}$ & 89 \\
6 & $1: 1.5$ & DCE & 90 \\
7 & $1: 1.5$ & EA & 87 \\
8 & $1: 1.5$ & $\mathrm{MeOH}$ & 85 \\
9 & $1: 1.5$ & EtOH & 86 \\
10 & $1: 1.5$ & DMSO & 79 \\
11 & $1: 1.5$ & DMF & 66 \\
$12^{c}$ & $1: 1.5$ & THF & 96
\end{tabular}

${ }^{a}$ Isolated yields. ${ }^{b}$ DCM: dichloromethane, THF: tetrahydrofuran, DCE: 1,2dichloroethane, EA: ethyl acetate; ${ }^{c}$ Under nitrogen.

\section{2 底物拓展}

在上述最优的反应条件下，对底物的普适性进行了 考察，具体结果见表 2 . 从实验结果可以看出，该方法 对各种取代的硫酚都有良好的普适性. 具体来说, 无论 是苯环上连有吸电子基 $[$ 氯 $(3 \mathbf{a}) 、$ 溴 $(3 \mathrm{~b})$ 、氟 $(3 \mathrm{c})]$ 还是给 电子基[甲基(3d)、甲氧基(3j)], 都能够以优秀的收率得 
到相应的一氟甲基二硫化合物. 同时，可能由于位阻的 影响, 苯环上邻位有取代基时收率明显降低. 比如 2-甲 基苯硫酚的收率为 $89 \%$ (3f), 邻甲氧基苯硫酚收率为 $64 \%(3 \mathbf{k}), 2,6$ 二甲基苯硫酚收率为 $62 \%(3 \mathrm{~g})$. 此外, 2-菜 硫酚也能以 $96 \%$ 的收率得到目标产物(3I). 杂芳环硫酚, 比如 2-颈基苯并噻唑和 2-颈基苯并噁唑, 在反应条件下 分别能以 $56 \%$ 和 $61 \%$ 的分离收率得到相应的产物( $3 \mathrm{~m}$, 3n).

表 2 硫酚类底物拓展 ${ }^{a, b}$

Table 2 Substrate scope for thiophenols

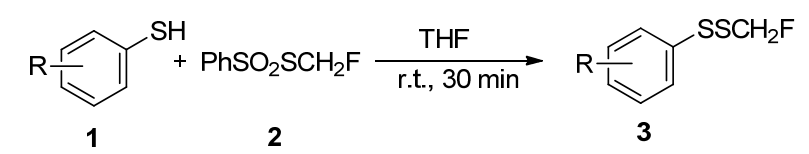

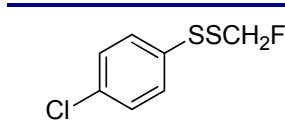

$3 a, 95 \%$<smiles>Cc1ccc(S(F)(F)CF)cc1</smiles>

3d, $92 \%$<smiles>Cc1cccc(C)c1S(F)(F)CF</smiles>

$3 \mathrm{~g}, 62 \%$

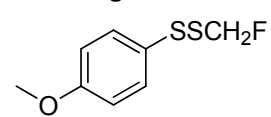

3j, $93 \%$

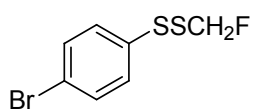

3b, $90 \%$<smiles>Cc1cccc(S(F)(F)F)c1</smiles>

$3 e, 93 \%$<smiles>Cc1ccc([SH](F)CF)c(C)c1</smiles>

3h, $89 \%$<smiles>COc1ccccc1S(F)(F)CF</smiles>

3k, $64 \%$

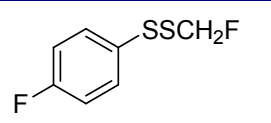

3c, $95 \%$

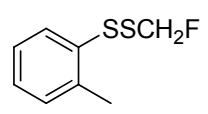

3f, $89 \%$

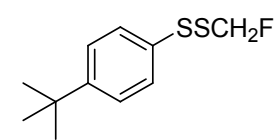

$3 \mathbf{i}, 73 \%$

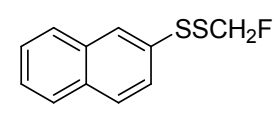

3I, $96 \%$<smiles>FCSc1nc2ccccc2s1</smiles>

$3 \mathrm{~m}, 56 \%$<smiles>FCSc1nc2ccccc2o1</smiles>

$3 n, 61 \%$
${ }^{a}$ Reaction conditions: thiophenol $(0.5 \mathrm{mmol})$ and $\mathrm{PhSO}_{2} \mathrm{SCH}_{2} \mathrm{~F}(0.75 \mathrm{mmol})$ in THF $(2 \mathrm{~mL})$ at r.t. for $30 \mathrm{~min} .{ }^{b}$ Yield of isolated product.

随后考察了硫醇底物对该反应条件的适用性. 如表 3 所示, 芐硫醇、4-氯芐硫醇和 2-苯基乙硫醇, 都能以优 秀的收率得到目标产物 $(\mathbf{4 a} \sim \mathbf{4 c})$. 长链的烷基硫醇, 比 如正十二烷基硫醇也能以 $80 \%$ 的收率得到相应的产物 (4f). 杂环硫醇, 比如 2-呋喃甲硫醇, 反应的收率仅为 $32 \%$ (4d), 而 2-吡嗪基乙硫醇, 则能以 $91 \%$ 的收率得到 产物(4g). 对于大位阻的三苯基甲硫醇，在该反应条件 下转化率较低，产物分离收率为 $29 \%$ (4e). 同时，对于 半胱氨酸衍生物, $N$-(叔丁氧基羰基)- $L$-半胱氨酸甲酯, 在该反应条件下也能以 $69 \%$ 的产率得到产物(4h).

接下来, 对该反应的机理进行了研究. 在之前的文 献报道中, $\mathrm{PhSO}_{2} \mathrm{SCH}_{2} \mathrm{~F}$ 对烯烃和酫的一氟甲硫基化反 应中涉及自由基历程 ${ }^{[8-9]}$. 我们小组 ${ }^{[10]}$ 报道了 $\mathrm{PhSO}_{2} \mathrm{~S}-$ $\mathrm{CH}_{2} \mathrm{~F}$ 和 $\mathrm{P}^{\mathrm{V}}-\mathrm{H}$ 化合物的一氟甲硫基化反应, 并提出了单
表 3 硫醇类底物拓展 ${ }^{a, b}$

Table 3 Substrate scope for mercaptans

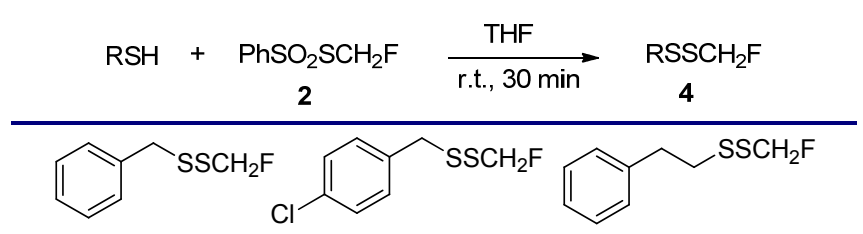

4a, $93 \%$

4b, $91 \%$

4c, $94 \%$

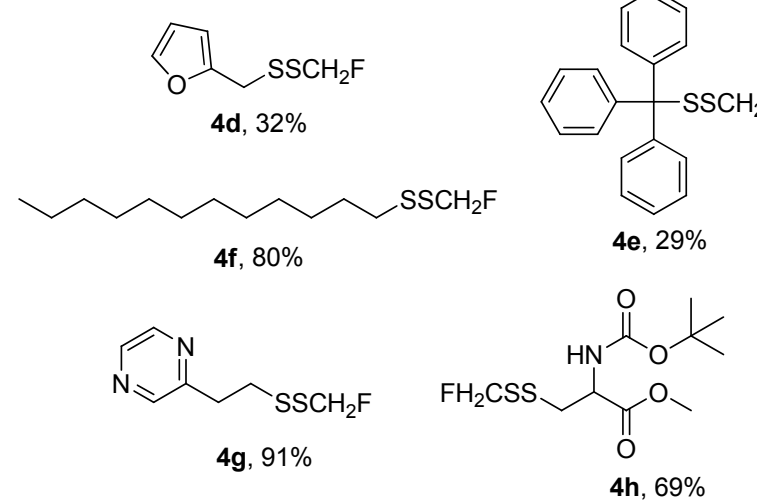

${ }^{a}$ Reaction conditions: mercaptan $(0.5 \mathrm{mmol})$ and $\mathrm{PhSO}_{2} \mathrm{SCH}_{2} \mathrm{~F}(0.75 \mathrm{mmol})$ in THF (2 mL) at r.t. for $30 \mathrm{~min} .{ }^{b}$ Yield of isolated product.

电子转移历程的可能反应机理. 为了验证该反应机理, 我们做了一系列控制实验. 在 4-氯苯硫酚和 $\mathrm{PhSO}_{2} \mathrm{~S}$ $\mathrm{CH}_{2} \mathrm{H}$ 的反应中, 分别加入自由基抑制剂 2,6-二叔丁基4-甲基苯酚(BHT)和四甲基哌啶氧化物(TEMPO), 一氟 甲基二硫化物的收率为 77\%和 53\%; 加入单电子捕获剂 1,3-二硝基苯和 1,4-二硝基苯，产物收率分别为 77\%和 $80 \%$. 实验结果表明，加入抑制剂该反应并没有得到明 显抑制, 因此该反应可能不是自由基历程. 由于 $\mathrm{PhSO}_{2} \mathrm{SCH}_{2} \mathrm{~F}$ 中存在容易断裂的 $\mathrm{S}-\mathrm{S}$ 键, 而硫酚(醇)的 硫原子上具有强的给电子能力, 可以作为软的亲核试剂 和 $\mathrm{PhSO}_{2} \mathrm{SCH}_{2} \mathrm{~F}$ 发生亲核取代反应. 因此, 我们推测该 反应机理可能为: 硫酚(醇)作为亲核试剂进攻 $\mathrm{PhSO}_{2} \mathrm{~S}$ $\mathrm{CH}_{2} \mathrm{~F}$ 中 $\mathrm{SCH}_{2} \mathrm{~F}$ 的硫原子, 形成质子化的中间体 $\mathbf{A}$, 苯 砜基 $\left(\mathrm{PhSO}_{2}\right)$ 带着一对电子离去; 中间体 $\mathbf{A}$ 进一步和苯 砜基负离子 $\left(\mathrm{PhSO}_{2}^{-}\right)$反应, 生成二硫化物 $\mathbf{B}$ 和苯亚磺酸 $\left(\mathrm{PhSO}_{2} \mathrm{H}\right)(\mathrm{Scheme})$. 进一步的机理研究仍在进行中.

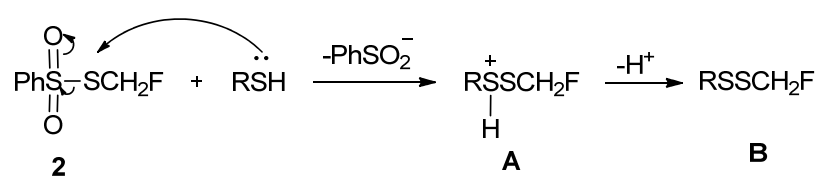

图式 2 可能的反应机理

Scheme 2 Proposed mechanism for the reaction

\section{2 结论}

综上所述，报道了在常温条件下以四氢呋喃为溶 
剂，从硫酚和硫醇出发高效合成一氟甲基二硫化物的方 法. 该方法具有反应条件温和、操作简单、反应速度快、 无需添加剂及产物收率高等优点, 为高效制备一氟甲硫 基二硫化物提供了一种新的方法.

\section{3 实验部分}

\section{1 仪器与试剂}

核磁共振氢谱、氟谱、碳谱的测定使用瑞士 Bruke 公司 $400 \mathrm{MHz}$ 傅立叶变换核磁共振波谱仪(AVANCE III)测定, 以 $\mathrm{TMS}$ 作内标, 気代氯仿为溶剂. 低分辨质 谱用 Agilent 5973N 型质谱仪测定. IR 使用 Thermo Nicolet IN10 MX 型红外分光光度仪测定, 固体用 $\mathrm{KBr}$ 压 片法, 液体用薄膜法. 熔点测试使用 WRS-1C 熔点仪.

\section{2 实验方法}

\subsection{1 $\mathrm{PhSO}_{2} \mathrm{SCH}_{2} \mathrm{~F}$ 试剂的合成}

在一个 $250 \mathrm{~mL}$ 的三口瓶中, 将 $\mathrm{PhSO}_{2} \mathrm{SNa}(9.81 \mathrm{~g}$, $50 \mathrm{mmol}$ ) 溶解在 $80 \mathrm{~mL}$ 的 $\mathrm{DMF}$ 中, 再缓慢溶解 $\mathrm{CH}_{2} \mathrm{ClF}$ (13.7 g, $200 \mathrm{mmol})$, 并在 $80{ }^{\circ} \mathrm{C}$ 下反应 $12 \mathrm{~h}$. 粗产品用二 氯甲烷和水萃取三次, 再用饱和食盐水洗涤一次, 收集 有机相, 用真空洜除去液相. 使用硅胶柱层析法 $[V($ 石油 醚) $: V$ (乙酸乙酯 $)=7: 1$ ] 得到无色液体产物 $\mathrm{PhSO}_{2} \mathrm{~S}-$ $\mathrm{CH}_{2} \mathrm{~F}^{[8 \mathrm{a}]} 4.0 \mathrm{~g}$, 产率 $40 \%$. ${ }^{1} \mathrm{H}$ NMR $\left(400 \mathrm{MHz}, \mathrm{CDCl}_{3}\right) \delta$ : $7.98 \sim 7.93(\mathrm{~m}, 2 \mathrm{H}), 7.66(\mathrm{t}, J=7.4 \mathrm{~Hz}, 1 \mathrm{H}), 7.57(\mathrm{t}, J=$ $7.6 \mathrm{~Hz}, 2 \mathrm{H}), 6.02(\mathrm{~d}, J=50.7 \mathrm{~Hz}, 2 \mathrm{H}) ;{ }^{19} \mathrm{~F} \mathrm{NMR}(376$ $\left.\mathrm{MHz}, \mathrm{CDCl}_{3}\right) \delta:-186.38$.

\section{2 .2 化合物 3 的合成}

在 $5 \mathrm{~mL}$ 的反应瓶中, 加入 $\mathrm{PhSO}_{2} \mathrm{SCH}_{2} \mathrm{~F}(154.5 \mathrm{mg}$, $0.75 \mathrm{mmol}$ )和 $2 \mathrm{~mL}$ 的四氢呋喃, 搅拌均匀后加入 4 -氯苯 硫酚(72.3 mg, $0.5 \mathrm{mmol}$ ), 在室温下反应 $30 \mathrm{~min}$. 粗产品 用真空泵除去溶剂, 使用硅胶柱层析法(石油醚)得到无 色液体产物 3a (97.8 mg, 95\%). 化合物 3b 3n 和 $\mathbf{4 a} \sim$ $4 \mathbf{h}$ 的合成方法同 $3 \mathbf{a}$.

1-(4-氯苯基)-2-(氟甲基)二硫醚 (3a)：无色液体， $95.4 \mathrm{mg}$, 产率 95\%. ${ }^{1} \mathrm{H} \mathrm{NMR}\left(300 \mathrm{MHz}, \mathrm{CDCl}_{3}\right) \delta: 7.52$ (d, $J=8.5 \mathrm{~Hz}, 2 \mathrm{H}), 7.30$ (d, $J=8.5 \mathrm{~Hz}, 2 \mathrm{H}), 5.66$ (d, $J=$ $51.7 \mathrm{~Hz}, 2 \mathrm{H}) ;{ }^{19} \mathrm{~F}$ NMR $\left(282 \mathrm{MHz}, \mathrm{CDCl}_{3}\right) \delta$ : -185.97 ; ${ }^{13} \mathrm{C}$ NMR (101 MHz, $\left.\mathrm{CDCl}_{3}\right) \delta: 135.24,134.17,130.73$, 129.41, 92.88 (d, $J=223.0 \mathrm{~Hz}$ ); HRMS (EI) calcd for $\mathrm{C}_{7} \mathrm{H}_{6} \mathrm{ClFS}_{2} 207.9583$, found 207.9592.

1-(4-溴苯基)-2-(氟甲基)二硫醚 (3b)：无色液体， $112.1 \mathrm{mg}$, 产率 90\%. ${ }^{1} \mathrm{H}$ NMR $\left(300 \mathrm{MHz}, \mathrm{CDCl}_{3}\right) \delta$ : $7.51 \sim 7.42(\mathrm{~m}, 4 \mathrm{H}), 5.66(\mathrm{~d}, J=54.3 \mathrm{~Hz}, 2 \mathrm{H}) ;{ }^{19} \mathrm{~F} \mathrm{NMR}$ $\left(282 \mathrm{MHz}, \mathrm{CDCl}_{3}\right) \delta:-186.08 ;{ }^{13} \mathrm{C} \mathrm{NMR}(101 \mathrm{MHz}$, $\left.\mathrm{CDCl}_{3}\right) \delta: 135.23(\mathrm{~d}, J=3.5 \mathrm{~Hz}), 131.67,130.14,121.45$,
92.21 (d, $J=223.1 \mathrm{~Hz}$ ); HRMS (EI) calcd for $\mathrm{C}_{7} \mathrm{H}_{6} \mathrm{BrFS}_{2}$ 251.9078 , found 251.9083 .

1-(4-氟苯基)-2-(氟甲基)二硫醚(3c)：无色液体, 77.8 $\mathrm{mg}$, 产率 95\%. ${ }^{1} \mathrm{H} \mathrm{NMR}\left(400 \mathrm{MHz}, \mathrm{CDCl}_{3}\right) \delta: 7.62 \sim 7.54$ $(\mathrm{m}, 2 \mathrm{H}), 7.04(\mathrm{t}, J=8.7 \mathrm{~Hz}, 2 \mathrm{H}), 5.67(\mathrm{~d}, J=51.8 \mathrm{~Hz}, 2 \mathrm{H})$; ${ }^{19} \mathrm{~F}$ NMR $\left(282 \mathrm{MHz}, \mathrm{CDCl}_{3}\right) \delta:-113.44,-186.11 ;{ }^{13} \mathrm{C}$ NMR (101 MHz, $\left.\mathrm{CDCl}_{3}\right) \delta: 162.82(\mathrm{~d}, J=248.7 \mathrm{~Hz})$, 132.39 (d, $J=8.4 \mathrm{~Hz}), 131.76,116.28$ (d, $J=22.2 \mathrm{~Hz})$, 92.72 (d, $J=222.6 \mathrm{~Hz}$ ); HRMS (EI) calcd for $\mathrm{C}_{7} \mathrm{H}_{6} \mathrm{~F}_{2} \mathrm{~S}_{2}$ 219.9358 , found 219.9359 .

1-(氟甲基)-2-(对甲苯基)二硫醚(3d):无色液体，85.6 $\mathrm{mg}$, 产率 92\%. ${ }^{1} \mathrm{H}$ NMR $\left(300 \mathrm{MHz}, \mathrm{CDCl}_{3}\right) \delta: 7.49(\mathrm{~d}$, $J=8.1 \mathrm{~Hz}, 2 \mathrm{H}), 7.15$ (d, $J=8.0 \mathrm{~Hz}, 2 \mathrm{H}), 5.66$ (d, $J=51.9$ $\mathrm{Hz}, 2 \mathrm{H}), 2.35$ (s, 3H); ${ }^{19} \mathrm{~F}$ NMR $\left(282 \mathrm{MHz}, \mathrm{CDCl}_{3}\right) \delta$ : $-186.32 ;{ }^{13} \mathrm{C} \mathrm{NMR}\left(101 \mathrm{MHz}, \mathrm{CDCl}_{3}\right) \delta: 138.30,132.95$, 130.15, 129.86, 92.62 (d, $J=222.2 \mathrm{~Hz}$ ), 21.06; HRMS (EI) calcd for $\mathrm{C}_{8} \mathrm{H}_{9} \mathrm{FS}_{2}$ 188.0130, found 188.0127.

1-(氟甲基)-2-(间甲苯基)二硫醚 $(3 e)^{[8 b]}$ : 淡黄色液 体, $90.1 \mathrm{mg}$, 产率 93\%. ${ }^{1} \mathrm{H} \mathrm{NMR}\left(400 \mathrm{MHz}, \mathrm{CDCl}_{3}\right) \delta$ : 7.38 (d, $J=6.7 \mathrm{~Hz}, 2 \mathrm{H}), 7.21$ (d, $J=12 \mathrm{~Hz}, 1 \mathrm{H}), 7.08$ (d, $J=7.3 \mathrm{~Hz}, 1 \mathrm{H}), 5.65(\mathrm{dd}, J=51.8,1.1 \mathrm{~Hz}, 2 \mathrm{H}), 2.35$ (s, $3 \mathrm{H}) ;{ }^{19} \mathrm{~F}$ NMR $\left(282 \mathrm{MHz}, \mathrm{CDCl}_{3}\right) \delta$ : $-186.32 ;{ }^{13} \mathrm{C} \mathrm{NMR}$ $\left(126 \mathrm{MHz}, \mathrm{CDCl}_{3}\right) \delta: 139.06,136.15,129.74,128.96$, 128.71, 126.27, 92.68 (d, $J=222.5 \mathrm{~Hz}$ ), 21.32; MS (EI) $m / z: 188.1\left(\mathrm{M}^{+}\right)$.

1-(氟甲基)-2-(邻甲苯基)二硫醚 $(\mathbf{3 f})^{[8 \mathrm{~b}]}$ : 淡黄色液 体, $86.7 \mathrm{mg}$, 产率 $89 \%$. ${ }^{1} \mathrm{H}$ NMR $\left(300 \mathrm{MHz}, \mathrm{CDCl}_{3}\right) \delta$ : $7.83 \sim 7.60(\mathrm{~m}, 1 \mathrm{H}), 7.24 \sim 7.18(\mathrm{~m}, 3 \mathrm{H}), 5.63(\mathrm{~d}, J=51.8$ $\mathrm{Hz}, 2 \mathrm{H}), 2.46(\mathrm{~s}, 3 \mathrm{H}) ;{ }^{19} \mathrm{~F}$ NMR $\left(282 \mathrm{MHz}, \mathrm{CDCl}_{3}\right) \delta$ : $-186.95 ;{ }^{13} \mathrm{C} \mathrm{NMR}\left(151 \mathrm{MHz}, \mathrm{CDCl}_{3}\right) \delta: 138.27,135.00$, 130.54, 130.40, 128.20, 126.80, 92.31 (d, $J=222.5 \mathrm{~Hz}$ ), 20.23; MS (EI) $m / z: 188.1\left(\mathrm{M}^{+}\right)$.

1-(2,6-二甲基苯基)-2-(氟甲基)二硫醚(3g)：淡黄色 液体, $60.9 \mathrm{mg}$, 产率 $62 \% .{ }^{1} \mathrm{H}$ NMR $\left(300 \mathrm{MHz} \mathrm{CDCl}_{3}\right) \delta$ : $7.21 \sim 7.06(\mathrm{~m}, 3 \mathrm{H}), 5.60(\mathrm{~d}, J=52.1 \mathrm{~Hz}, 2 \mathrm{H}), 2.59$ (s, $6 \mathrm{H}) ;{ }^{19} \mathrm{~F}$ NMR $\left(282 \mathrm{MHz}, \mathrm{CDCl}_{3}\right) \delta$ : $-186.96 ;{ }^{13} \mathrm{C} \mathrm{NMR}$ $\left(101 \mathrm{MHz}, \mathrm{CDCl}_{3}\right) \delta: 142.81,134.20,129.64,128.50$, 91.09 (d, $J=222.3 \mathrm{~Hz}), 21.94$; IR (KBr) v: 3395, 2922, 2848, 1941, 1646, 1582, 1461, 1421, 1376, 1311, 1245, 1167, 983, 773, 710, $421 \mathrm{~cm}^{-1}$; HRMS (EI) calcd for $\mathrm{C}_{9} \mathrm{H}_{11} \mathrm{FS}_{2}$ 202.0286, found 202.0293.

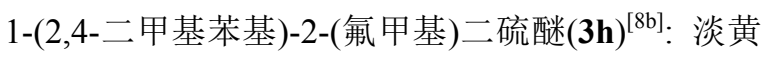
色液体, $89.0 \mathrm{mg}$, 产率 $89 \% .{ }^{1} \mathrm{H} \mathrm{NMR}\left(400 \mathrm{MHz}, \mathrm{CDCl}_{3}\right)$ $\delta: 7.57(\mathrm{~d}, J=7.8 \mathrm{~Hz}, 1 \mathrm{H}), 7.05 \sim 6.99(\mathrm{~m}, 2 \mathrm{H}), 5.61(\mathrm{~d}$, $J=51.9 \mathrm{~Hz}, 2 \mathrm{H}), 2.44(\mathrm{~s}, 3 \mathrm{H}), 2.31(\mathrm{~s}, 3 \mathrm{H}) ;{ }^{19} \mathrm{~F}$ NMR $(282$ 
$\left.\mathrm{MHz}, \mathrm{CDCl}_{3}\right) \delta:-187.03 ;{ }^{13} \mathrm{C} \mathrm{NMR}\left(101 \mathrm{MHz}, \mathrm{CDCl}_{3}\right)$ $\delta: 139.08,138.91,131.97,131.96,131.48,127.55,92.15$ (d, $J=222.3 \mathrm{~Hz}$ ), 21.03, 20.34; MS (EI) $\mathrm{m} / z$ : $202.1\left(\mathrm{M}^{+}\right)$.

1-(4-(叔丁基)苯基)-2-(氟甲基)二硫醚(3i): 淡黄色液 体, $83.3 \mathrm{mg}$, 产率 $73 \% .{ }^{1} \mathrm{H} \mathrm{NMR}\left(400 \mathrm{MHz}, \mathrm{CDCl}_{3}\right) \delta$ : $7.55 \sim 7.50(\mathrm{~m}, 2 \mathrm{H}), 7.39 \sim 7.34(\mathrm{~m}, 2 \mathrm{H}), 5.66(\mathrm{~d}, J=51.8$ $\mathrm{Hz}, 2 \mathrm{H}), 1.31$ (s, 9H); ${ }^{19} \mathrm{~F} \mathrm{NMR}\left(376 \mathrm{MHz}, \mathrm{CDCl}_{3}\right) \delta$ : $-185.85 ;{ }^{13} \mathrm{C}$ NMR $\left(151 \mathrm{MHz}, \mathrm{CDCl}_{3}\right) \delta: 151.49,132.97$, 129.71, 126.26, 92.73 (d, $J=222.3 \mathrm{~Hz}), 34.64,31.24$; HRMS (EI) calcd for $\mathrm{C}_{11} \mathrm{H}_{15} \mathrm{FS}_{2}$ 230.0599, found 230.0594 .

1-(氟甲基)-2-(4-甲氧基苯基)二硫醚 $(\mathbf{3} \mathbf{j})$ ：黄色液体， $100.1 \mathrm{mg}$, 产率 93\%. ${ }^{1} \mathrm{H} \mathrm{NMR}\left(400 \mathrm{MHz}, \mathrm{CDCl}_{3}\right) \delta: 7.54$ $(\mathrm{d}, J=8.5 \mathrm{~Hz}, 2 \mathrm{H}), 6.86(\mathrm{~d}, J=8.5 \mathrm{~Hz}, 2 \mathrm{H}), 5.66(\mathrm{~d}, J=$ $52.0 \mathrm{~Hz}, 2 \mathrm{H}), 3.80(\mathrm{~s}, 3 \mathrm{H}) ;{ }^{19} \mathrm{~F}$ NMR $\left(282 \mathrm{MHz}, \mathrm{CDCl}_{3}\right) \delta$ : $-186.30 ;{ }^{13} \mathrm{C}$ NMR (101 MHz, $\left.\mathrm{CDCl}_{3}\right) \delta: 160.29,133.50$, 127.17, 114.76, 92.56 (d, $J=221.9 \mathrm{~Hz}), 55.36$; HRMS (EI) calcd for $\mathrm{C}_{8} \mathrm{H}_{9} \mathrm{FOS}_{2} 204.0079$, found 204.0084.

1-(氟甲基)-2-(2-甲氧基苯基)二硫醚(3k): 黄色液 体, $65.7 \mathrm{mg}$, 产率 64\%. ${ }^{1} \mathrm{H} \mathrm{NMR}\left(400 \mathrm{MHz}, \mathrm{CDCl}_{3}\right) \delta$ : $7.69(\mathrm{~d}, J=8.4 \mathrm{~Hz}, 1 \mathrm{H}), 7.31 \sim 7.27(\mathrm{~m}, 1 \mathrm{H}), 6.98(\mathrm{t}, J=$ $7.6 \mathrm{~Hz}, 1 \mathrm{H}), 6.89$ (d, $J=8.2 \mathrm{~Hz}, 1 \mathrm{H}), 5.67$ (d, $J=51.8 \mathrm{~Hz}$, 2H), $3.91(\mathrm{~s}, 3 \mathrm{H}) ;{ }^{19} \mathrm{~F}$ NMR (376 MHz, $\left.\mathrm{CDCl}_{3}\right) \delta$ : $-185.91 ;{ }^{13} \mathrm{C}$ NMR (151 MHz, $\left.\mathrm{CDCl}_{3}\right) \delta: 157.42,130.55$, 129.35, 124.33, 121.24, 110.98, 92.85 (d, $J=221.5 \mathrm{~Hz})$, 55.93; IR (KBr) v: 2924, 2837, 1579, 1474, 1433, 1311, 1272, 1240, 1181, 1162, 1129, 1059, 1020, 974, 789, 747, $706 \mathrm{~cm}^{-1}$; HRMS (EI) calcd for $\mathrm{C}_{8} \mathrm{H}_{9} \mathrm{FOS}_{2} 204.0079$, found 204.0085 .

1-(氟甲基)-2-(菜-2-基)二硫醚(31):白色固体，107.7 $\mathrm{mg}$, 产率 96\%. m.p. $42 \sim 44{ }^{\circ} \mathrm{C} ;{ }^{1} \mathrm{H}$ NMR $(400 \mathrm{MHz}$, $\left.\mathrm{CDCl}_{3}\right) \delta: 8.05(\mathrm{~s}, 1 \mathrm{H}), 7.85 \sim 7.78(\mathrm{~m}, 3 \mathrm{H}), 7.66(\mathrm{dd}, J=$ 8.6, $1.9 \mathrm{~Hz}, 1 \mathrm{H}), 7.55 \sim 7.44(\mathrm{~m}, 2 \mathrm{H}), 5.69(\mathrm{~d}, J=51.7 \mathrm{~Hz}$, $2 \mathrm{H}) ;{ }^{19} \mathrm{~F}$ NMR $\left(376 \mathrm{MHz}, \mathrm{CDCl}_{3}\right) \delta:-185.99 ;{ }^{13} \mathrm{C} \mathrm{NMR}$ $\left(101 \mathrm{MHz}, \mathrm{CDCl}_{3}\right) \delta: 133.11(\mathrm{~d}, J=3.2 \mathrm{~Hz}), 132.94$, $132.23,128.61,127.72,127.35,127.15,126.39,126.27$, 126.11, 92.11 (d, $J=222.9 \mathrm{~Hz}$ ); IR (KBr) v: 3054, 2950, 2920, 2848, 1646, 1586, 1499, 1422, 1337, 1314, 1267, 1197, 1131, 1081, 1001, 976, 816, 737, 699, 628, 477, 466 $\mathrm{cm}^{-1}$; HRMS (EI) calcd for $\mathrm{C}_{11} \mathrm{H}_{9} \mathrm{FS}_{2} 224.0130$, found 224.0123 .

2-((氟甲基)二硫基)苯并噻唑 $(\mathbf{3 m})$ ：深黄色液体， $65.7 \mathrm{mg}$, 产率 56\%. ${ }^{1} \mathrm{H}$ NMR (400 $\left.\mathrm{MHz}, \mathrm{CDCl}_{3}\right) \delta: 7.91$ $(\mathrm{d}, J=8.1 \mathrm{~Hz}, 1 \mathrm{H}), 7.82(\mathrm{~d}, J=7.9 \mathrm{~Hz}, 1 \mathrm{H}), 7.46$ (t, $J=7.4$ $\mathrm{Hz}, 1 \mathrm{H}), 7.36$ (t, $J=7.4 \mathrm{~Hz}, 1 \mathrm{H}), 5.78$ (d, $J=51.0 \mathrm{~Hz}, 2 \mathrm{H})$;
${ }^{19} \mathrm{~F}$ NMR $\left(282 \mathrm{MHz}, \mathrm{CDCl}_{3}\right) \delta:-187.12 ;{ }^{13} \mathrm{C}$ NMR (151 $\left.\mathrm{MHz}, \mathrm{CDCl}_{3}\right) \delta: 169.57,154.59,136.09,126.46,125.02$, 122.46, 121.18, 91.62 (d, $J=226.1 \mathrm{~Hz})$; IR (KBr) v: 3061, 2926, 2850, 1460, 1425, 1312, 1237, 1082, 1020, 1007, $757,727,703,700,430 \mathrm{~cm}^{-1}$; HRMS (EI) calcd for $\mathrm{C}_{8} \mathrm{H}_{6}$ $\mathrm{NFS}_{3} 230.9646$, found 230.9654 .

2-((氟甲基)二硫基)苯并噁唑 $(3 n)$ : 淡黄色液体， $65.6 \mathrm{mg}$, 产率 $61 \%$. ${ }^{1} \mathrm{H} \mathrm{NMR}\left(400 \mathrm{MHz}, \mathrm{CDCl}_{3}\right) \delta$ : $7.76 \sim 7.65(\mathrm{~m}, 1 \mathrm{H}), 7.57 \sim 7.48(\mathrm{~m}, 1 \mathrm{H}), 7.40 \sim 7.31(\mathrm{~m}$, 2H), $5.81(\mathrm{~d}, J=51.1 \mathrm{~Hz}, 2 \mathrm{H}) ;{ }^{19} \mathrm{~F}$ NMR (376 MHz, $\left.\mathrm{CDCl}_{3}\right) \delta:-188.68 ;{ }^{13} \mathrm{C} \mathrm{NMR} \delta: 161.76,152.54,141.79$, 125.24, 124.86, 119.65, 110.46, 90.99 (d, $J=225.5 \mathrm{~Hz}$ ); IR (KBr) v: 3049, 2927, 1774, 1496, 1450, 1314, 1237, 1217, 1129, 1093, 988, 923, 801, 743, $594 \mathrm{~cm}^{-1}$; HRMS (EI) calcd for $\mathrm{C}_{8} \mathrm{H}_{6} \mathrm{FNOS}_{2} 214.9875$, found 214.9872 .

1-苄基-2-(氟甲基)二硫醚(4a)：淡黄色液体，88.6 $\mathrm{mg}$, 产率 93\%. ${ }^{1} \mathrm{H} \mathrm{NMR}\left(300 \mathrm{MHz}, \mathrm{CDCl}_{3}\right) \delta: 7.36 \sim 7.28$ $(\mathrm{m}, 5 \mathrm{H}), 5.41(\mathrm{~d}, J=51.9 \mathrm{~Hz}, 2 \mathrm{H}), 4.04(\mathrm{~s}, 2 \mathrm{H}) ;{ }^{19} \mathrm{~F}$ NMR $\left(282 \mathrm{MHz}, \mathrm{CDCl}_{3}\right) \delta$ : $-185.88 ;{ }^{13} \mathrm{C} \mathrm{NMR}(101 \mathrm{MHz}$, $\left.\mathrm{CDCl}_{3}\right) \delta: 137.00(\mathrm{~d}, J=66.4 \mathrm{~Hz}), 129.36,128.49$ (d, $J=$ $11.5 \mathrm{~Hz}), 127.50$ (d, $J=25.7 \mathrm{~Hz}), 93.36$ (d, $J=219.6 \mathrm{~Hz}$ ), $43.88(\mathrm{~d}, J=124.7 \mathrm{~Hz})$; IR $(\mathrm{KBr}) v: 3062,3030,2927$, 2854, 1948, 1601, 1495, 1454, 1420, 1313, 1232, 1200, 1071, 1028, 979, 756, 699, 564, $471 \mathrm{~cm}^{-1}$; HRMS (EI) calcd for $\mathrm{C}_{8} \mathrm{H}_{9} \mathrm{FS}_{2} 188.0130$, found 188.0136.

1-(4-氯苄基)-2-(氟甲基)二硫醚(4b): 无色液体， $82.6 \mathrm{mg}$, 产率 91\%. ${ }^{1} \mathrm{H} \mathrm{NMR}\left(400 \mathrm{MHz}, \mathrm{CDCl}_{3}\right) \delta$ : $7.33 \sim 7.28(\mathrm{~m}, 2 \mathrm{H}), 7.27 \sim 7.23(\mathrm{~m}, 2 \mathrm{H}), 5.47(\mathrm{~d}, J=51.9$ $\mathrm{Hz}, 2 \mathrm{H}), 3.99$ (s, 2H); ${ }^{19} \mathrm{~F}$ NMR (376 MHz, $\left.\mathrm{CDCl}_{3}\right) \delta$ : $-185.33 ;{ }^{13} \mathrm{C}$ NMR (101 MHz, $\left.\mathrm{CDCl}_{3}\right) \delta: 135.50$ (d, $J=$ $54.3 \mathrm{~Hz}), 133.45$ (d, $J=17.7 \mathrm{~Hz}), 130.64$ (d, $J=7.9 \mathrm{~Hz}$ ), 128.67 (d, $J=7.7 \mathrm{~Hz}), 93.49$ (d, $J=219.7 \mathrm{~Hz}), 43.07$ (d, $J=121.6 \mathrm{~Hz}$ ); IR (KBr) v: 3397, 3028, 2925, 2850, 1897, 1596, 1418, 1405, 1312, 1229, 1199, 1093, 1016, 980, 833, $808,736,638,504 \mathrm{~cm}^{-1}$; HRMS (EI) calcd for $\mathrm{C}_{8} \mathrm{H}_{8} \mathrm{FClS}_{2}$ 221.9740 , found 221.9746 .

1-(氟甲基)-2-苯乙基二硫醚(4c):黄色液体, $97.3 \mathrm{mg}$, 产率 94\%. ${ }^{1} \mathrm{H}$ NMR (400 $\left.\mathrm{MHz}, \mathrm{CDCl}_{3}\right) \delta: 7.30(\mathrm{t}, J=7.2$ $\mathrm{Hz}, 2 \mathrm{H}), 7.24 \sim 7.18(\mathrm{~m}, 3 \mathrm{H}), 5.61(\mathrm{~d}, J=52.0 \mathrm{~Hz}, 2 \mathrm{H})$, $3.10 \sim 3.05(\mathrm{~m}, 2 \mathrm{H}), 3.05 \sim 2.99(\mathrm{~m}, 2 \mathrm{H}) ;{ }^{19} \mathrm{~F}$ NMR $(376$ $\left.\mathrm{MHz}, \mathrm{CDCl}_{3}\right) \delta:-188.10 ;{ }^{13} \mathrm{C} \mathrm{NMR}\left(101 \mathrm{MHz}, \mathrm{CDCl}_{3}\right)$ $\delta: 139.67,128.60,128.51,126.45,93.83$ (d, $J=219.8 \mathrm{~Hz})$, 41.24, 35.48; IR (KBr) v: 3027, 2960, 2924, 2850, 1724, 1603, 1496, 1453, 1312, 1262, 1072, 1029, 977, 799, 748, 698, 564, $490 \mathrm{~cm}^{-1}$; HRMS (EI) calcd for $\mathrm{C}_{9} \mathrm{H}_{11} \mathrm{FS}_{2}$ 
202.0286, found 202.0293.

2-((氟甲基)二硫基)甲基呋喃(4d): 无色液体，35.1 $\mathrm{mg}$, 产率 32\%. ${ }^{1} \mathrm{H} \mathrm{NMR}\left(400 \mathrm{MHz}, \mathrm{CDCl}_{3}\right) \delta: 7.41 \sim 7.38$ $(\mathrm{m}, 1 \mathrm{H}), 6.35 \sim 6.32(\mathrm{~m}, 1 \mathrm{H}), 6.29(\mathrm{~d}, J=3.2 \mathrm{~Hz}, 1 \mathrm{H})$, $5.42(\mathrm{~d}, J=51.8 \mathrm{~Hz}, 2 \mathrm{H}), 4.04(\mathrm{~s}, 2 \mathrm{H}) ;{ }^{19} \mathrm{~F}$ NMR $(376$ $\left.\mathrm{MHz}, \mathrm{CDCl}_{3}\right) \delta:-185.80 ;{ }^{13} \mathrm{C} \mathrm{NMR}\left(151 \mathrm{MHz}, \mathrm{CDCl}_{3}\right)$ $\delta: 149.69,142.77,110.66,109.27,93.30$ (d, $J=219.1 \mathrm{~Hz})$, 36.71; IR (KBr) v: 2929, 1591, 1501, 1421, 1404, 1313, 1241, 1209, 1150, 1011, 973, 935, 884, 810, 737, 707, 598, $\mathrm{cm}^{-1}$; HRMS (EI) calcd for $\mathrm{C}_{6} \mathrm{H}_{7} \mathrm{FOS}_{2}$ 177.9922, found 177.9925

1-(氟甲基)-2-三苯甲基二硫醚(4e)：黄红色固体， $49.3 \mathrm{mg}$, 产率 29\%. m.p. 39 41 ${ }^{\circ} \mathrm{C}$; ${ }^{1} \mathrm{H}$ NMR (400 MHz, $\left.\mathrm{CDCl}_{3}\right) \delta: 7.43(\mathrm{~d}, J=7.5 \mathrm{~Hz}, 6 \mathrm{H}), 7.34 \sim 7.26(\mathrm{~m}, 8 \mathrm{H})$, $7.26 \sim 7.21(\mathrm{~m}, 1 \mathrm{H}), 4.51(\mathrm{~d}, J=51.8 \mathrm{~Hz}, 2 \mathrm{H}) ;{ }^{19} \mathrm{~F}$ NMR $\left(376 \mathrm{MHz}, \mathrm{CDCl}_{3}\right) \delta:-187.52 ;{ }^{13} \mathrm{C} \mathrm{NMR}(151 \mathrm{MHz}$, $\left.\mathrm{CDCl}_{3}\right) \delta: 143.22,129.98,128.00,127.20,89.83(\mathrm{~d}, J=$ $219.6 \mathrm{~Hz}), 71.70$; IR (KBr) v: 2924, 2855, 1489, 1441, 1303, 1184, 1029, 966, 757, 733, 695, 626, 527, $489 \mathrm{~cm}^{-1}$; MS (EI) $m / z: 243(100)\left[\mathrm{M}-\mathrm{SSCH}_{2} \mathrm{~F}\right]^{+}$.

1-十二烷基-2-(氟甲基)二硫醚(4f)：无色液体，108.8 $\mathrm{mg}$, 产率 80\%. ${ }^{1} \mathrm{H}$ NMR (400 MHz, $\left.\mathrm{CDCl}_{3}\right) \delta: 5.58$ (d, $J=52.1 \mathrm{~Hz}, 2 \mathrm{H}), 2.84 \sim 2.78(\mathrm{~m}, 2 \mathrm{H}), 2.54 \sim 2.47(\mathrm{~m}, 2 \mathrm{H})$, $1.68(\mathrm{dt}, J=14.8,7.1 \mathrm{~Hz}, 2 \mathrm{H}), 1.59$ (dt, $J=14.8,7.2 \mathrm{~Hz}$, $2 \mathrm{H}), 1.36 \sim 1.18(\mathrm{~m}, 14 \mathrm{H}), 0.89 \sim 0.84(\mathrm{~m}, 3 \mathrm{H}) ;{ }^{19} \mathrm{~F}$ NMR $\left(376 \mathrm{MHz}, \mathrm{CDCl}_{3}\right) \delta$ : $-188.51 ;{ }^{13} \mathrm{C} \mathrm{NMR}(151 \mathrm{MHz}$, $\left.\mathrm{CDCl}_{3}\right) \delta: 93.91(\mathrm{~d}, J=220.0 \mathrm{~Hz}), 40.21,34.07,31.92$, $29.64,29.60,29.54,29.36,29.09,28.40,24.65,22.69$, 14.10; IR (KBr) v: 2924, 2853, 1465, 1377, 1311, 984, 711 $\mathrm{cm}^{-1}$; HRMS (EI) calcd for $\mathrm{C}_{13} \mathrm{H}_{27} \mathrm{FS}_{2} 266.1538$, found 266.1541 .

2-(2-((氟甲基)二硫基)乙基)吡嗪 $(\mathbf{4 g})$ ：淡黄色液体， $92.8 \mathrm{mg}$, 产率 91\%. ${ }^{1} \mathrm{H}$ NMR (400 $\left.\mathrm{MHz}, \mathrm{CDCl}_{3}\right) \delta$ : $8.54 \sim 8.52(\mathrm{~m}, 1 \mathrm{H}), 8.51(\mathrm{~s}, 1 \mathrm{H}), 8.46 \sim 8.43(\mathrm{~m}, 1 \mathrm{H})$, $5.62(\mathrm{~d}, J=52.0 \mathrm{~Hz}, 2 \mathrm{H}), 3.26(\mathrm{~s}, 4 \mathrm{H}) ;{ }^{19} \mathrm{~F}$ NMR $(376$ $\left.\mathrm{MHz}, \mathrm{CDCl}_{3}\right) \delta:-184.09 ;{ }^{13} \mathrm{C} \mathrm{NMR}\left(101 \mathrm{MHz}, \mathrm{CDCl}_{3}\right)$ $\delta: 154.96,144.97,144.23,142.75,93.68$ (d, $J=220.4 \mathrm{~Hz})$, 38.37, 34.45; IR (KBr) v: 3066, 2923, 1579, 1527, 1475, 1404, 1312, 1231, 1159, 1108, 1059, 967, 831, 704, 406 $\mathrm{cm}^{-1}$; MS (EI) $m / z: 139(100)\left[\mathrm{M}-\mathrm{SCH}_{2} \mathrm{~F}\right]^{+}$.

$N$-(叔丁氧羰基)- $S$-(氟烷硫基)半胱氨酸甲酯(4h): 无色液体, $103.1 \mathrm{mg}$, 产率 69\%. ${ }^{1} \mathrm{H}$ NMR (400 MHz, $\left.\mathrm{CDCl}_{3}\right) \delta: 5.62(\mathrm{~d}, J=51.8 \mathrm{~Hz}, 2 \mathrm{H}), 4.60(\mathrm{~s}, 1 \mathrm{H}), 3.78(\mathrm{~s}$, $3 \mathrm{H}), 3.41 \sim 3.10(\mathrm{~m}, 2 \mathrm{H}), 1.46(\mathrm{~s}, 9 \mathrm{H}) ;{ }^{19} \mathrm{~F}$ NMR $(376$ $\left.\mathrm{MHz}, \mathrm{CDCl}_{3}\right) \delta:-185.79 ;{ }^{13} \mathrm{C}$ NMR $\left(101 \mathrm{MHz}, \mathrm{CDCl}_{3}\right)$ $\delta: 171.09,155.03,93.38(\mathrm{~d}, J=219.9 \mathrm{~Hz}), 80.32,53.43$, 52.64, 42.44; IR (KBr) v: 3328, 2976, 1695, 1512, 1435, 1368, 1313, 1248, 1162, 1047, 981, 880, $708 \mathrm{~cm}^{-1}$; HRMS (EI) calcd for $\mathrm{C}_{10} \mathrm{H}_{18} \mathrm{FNO}_{4} \mathrm{~S}_{2} 299.0661$, found 299.0660.

辅助材料(Supporting Information) 化合物 $\mathbf{3 a} \sim \mathbf{3 n}$ 和 4a $\sim \mathbf{4 h}$ 及 $\mathrm{PhSO}_{2} \mathrm{SCH}_{2} \mathrm{~F}$ 的 ${ }^{1} \mathrm{H} \mathrm{NMR},{ }^{19} \mathrm{~F} \mathrm{NMR},{ }^{13} \mathrm{C} \mathrm{NMR}$ 图谱。这些材料可以免费从本刊网站 (http://siocjournal.cn/)上下载.

\section{References}

[1] (a) Zhang, P. P.; Lu, L.; Shen, Q. L. Acta Chim. Sinica 2017, 75, 744 (in Chinese).

(张盼盼, 吕龙, 沈其龙, 化学学报, 2017, 75, 744.)

(b) Geng, Y.; Liang, A. P.; Gao, X. Y.; Niu, C. S.; Li, J. Y.; Zou, D. P; Wu, Y. S.; Wu, Y. J. J. Org. Chem. 2017, 82, 8604.

[2] (a) Yang, X. Y.; Wu, T.; Phipps, R. J.; Toste, F. D. Chem. Rev. 2015 115,826 .

(b) Yan, Q.; Jiang, L. P.; Yi, W. B. Chin. J. Org. Chem. 2020, 40, 1 (in Chinese). (间强，蒋绿齐，易文斌，有机化学, 2020, 40, 1.)

[3] (a) Leroux, F.; Jeschke, P.; Schlosser, M. Chem. Rev. 2005, 105,827 .

(b) Boiko, V. N. Beilstein J. Org. Chem. 2010, 6, 880

(c) He, W. M.; Weng, Z. Q. Prog. Chem. 2013, 25, 1071 (in Chinese).

(何伟明，翁志强，化学进展, 2013, 25, 1071.)

(d) Wang, Z.; Tu, Q.; Weng, Z. J. Organomet. Chem. 2014, 751, 830

(e) Nikolaienko, P.; Pluta, R.; Rueping, M. Chem.-Eur. J. 2014, 20, 9867.

(f) Li, S. S.; Wang, J. B. Acta Chim. Sinica 2018, 76, 913 (in Chinese).

(李树森，王剑波，化学学报, 2018, 76, 913.)

(g) Yang, Y. D.; Azuma, A.; Tokunaga, E.; Yamasaki, M.; Shiro, M.; Shibata, N. J. Am. Chem. Soc. 2013, 135, 8782.

(h) Shao, X. X.; Wang, X. Q.; Yang, T.; Lu, L.; Shen, Q. L. Angew Chem., Int. Ed. 2013, 52, 1.

(i) Zhu, D. H.; Shao, X. X.; Hong, X.; Lu, L.; Shen, Q. L. Angew. Chem., Int. Ed. 2016, 55, 15807.

[4] (a) Zhao, X.; Li, T. J.; Yang, B.; Qiu. D.; Lu, K. Tetrahedron 2017 73,3112 .

(b) Ismalaj, E.; Billard, T. J. Fluorine Chem. 2017, 203, 215.

(c) Xu, B.; Wang, D. C.; Hu, Y. H.; Shen, Q. L. Org. Chem. Front. 2018, 5,1462 .

[5] (a) Howland W. C. Clin. Exp Allergy 1996, 26, 18. (b) Zhou, J.; Jin, C.; Su, W. Org. Process Res. Dev. 2014, 18, 928.

[6] Ashton, M. J.; Lawrence, C.; Karlsson, J. A.; Stuttle, K. A. J.; Newton, C. G.; Vacher, B. Y. J.; Webber, S.; Withnall, M. J. J. Med. Chem. 1996, 39, 4888.

[7] Jung, M.; Wahl, A. F.; Neupert, W.; Geisslinger, G.; Senter, P. D. Pharm. Pharmacol. Commun. 2000, 6, 217.

[8] (a) Zhao, Q. C.; Lu, L.; Shen, Q. L. Angew. Chem. 2017, 129, 11733.

(b) Liu, F. M.; Jiang, L. Q.; Qiu, H. Y.; Yi, W. B. Org. Lett. 2018 , 20, 6270 .

[9] (a) Guo, S. H.; Wang, M. Y.; Pan, G. F.; Zhu, X. Q.; Gao, Y. R.; Wang, Y. Q. Adv. Synth. Catal. 2018, 360, 1861. (b) Xu, B.; Li, D. Z.; Lu, L.; Wang, D. C.; Hu, Y. H.; Shen, Q. L. Org. Chem. Front. 2018, 5, 2163.

[10] Pang, R. Y.; Yao, R. C.; Lu, S. Y.; Zhou, Y. C.; Chen, W. B. Chin. Chem. Lett. 2021, 32, 453.

[11] Wang, W. G.; Lin, Y. Z.; Ma, Y. D.; Tung, C. H.; Xu, Z. H. Org. 
Lett. 2018, 20, 3829

[12] (a) Cheng, Z.; Zhang, J.; Ballou, D. P.; Williams, C. H., Jr. Chem. Rev. 2011, 111,5768

(b) Block, E.; Bayer, T.; Naganathan, S.; Zhao, S. H. J. Am. Chem. Soc. 1996, 118, 2799.

(c) Chankhamjon, P.; Boettger-Schmidt, D.; Scherlach, K.; Urbansky, B.; Lackner, G.; Kalb, D.; Dahse, H.-M.; Hoffmeister, D.; Hertweck, C. Angew. Chem., Int. Ed. 2014, 53, 13409.

(d) Xiao, X.; Xue, J.; Jiang, X. Nat. Commun. 2018, 9, 2191.

[13] (a) Sonia, T. V.; Erben, M. F.; Boese R.; Vedova, C. O. New J.
Chem. 2010, 34, 1365.

(b) Pluta, R.; Rueping, M. Chem.-Eur. J. 2014, 20, 17315.

(c) Glenadel, Q.; Billard, T. Chin. J. Chem. 2016, 34, 455.

[14] (a) Xu, C.; Ma, B.; Shen, Q. L. Angew. Chem., Int. Ed. 2014, 53, 9316.

(b) Zhu, D. H.; Gu, Y.; Lu, L.; Shen, Q. L. J. Am. Chem. Soc. 2015, $137,10547$.

[15] Huang, P. F.; Wang, P.; Tang, S.; Fu, Z. J.; Lei, A. W. Angew. Chem. 2018, 130,8247 